\title{
Circadian Rhythm Variations and Nutrition in Children
}

\author{
Marie Gombert ${ }^{1,2}$ Joaquín Carrasco-Luna ${ }^{1,3}$ Gonzalo Pin-Arboledas ${ }^{4}$ Pilar Codoñer-Franch ${ }^{1,5}$
}

${ }^{1}$ Department of Pediatrics, Obstetrics, and Gynecology, University of Valencia, Valencia, Spain

2 Département de Biotechnologies, Université de La Rochelle, La Rochelle, France

${ }^{3}$ Department of Experimental Science, School Catholic University of Address for correspondence Marie Gombert, MSc, Department of Pediatrics, Obstetrics, and Gynecology, University of Valencia, Avenida de Blasco Ibáñez 15, 46010 Valencia, Spain

Valencia, Valencia, Spain

${ }^{4}$ Department of Pediatrics, Pediatric Sleep Unit, Hospital Quironsalud Hospital, Valencia, Spain

${ }^{5}$ Department of Pediatrics, Dr Peset University Hospital, Valencia, Spain (e-mail: marie_gombert@orange.fr).

J Child Sci 2018;8:e60-e66.

\begin{abstract}
Keywords

- circadian rhythms

- nutrition

- children

- chronodisruption

- metabolic disorders

Circadian rhythms are the changes in biological processes that occur on a daily basis. Among these processes are reactions involved in metabolic homeostasis. Circadian rhythms are structured by the central clock in the suprachiasmatic nucleus of the hypothalamus via the control of melatonin expression. Circadian rhythms are also controlled by the peripheral clocks, which are intracellular mechanisms composed of the clock genes, whose expression follows a circadian pattern. Circadian rhythms are impacted by signals from the environment called zeitgebers, or time givers, which include light exposure, feeding schedule and composition, sleeping schedule and pattern, temperature, and physical exercise. When the signals from the environment are synchronized with the internal clocks, metabolism is optimized. The term chronodisruption is used to describe the opposite situation. The latest research has demonstrated that life habits coherent with the internal clocks should be adopted, especially during childhood, to prevent metabolic diseases. Nevertheless, a few studies have investigated this link in children, and key information remains unknown.
\end{abstract}

\section{Introduction}

The expression circadian rhythm comes from the Latin circā ("about") and diēs ("a day"). This expression is used to define changes in biological processes that occur on a daily basis. Endogenous circadian clocks regulate 24-hour rhythms of behavior and physiology to align with external time. ${ }^{1} \mathrm{~A}$ vast number of vital reactions display a circadian rhythm, such as body temperature, blood pressure, and heart rate as well as the expression of numerous hormones and inflammatory factors.

In the first part of this review, we describe the mechanisms involved in the structure and regulation of circadian rhythms: the role of melatonin, the main circadian hormone; the clock genes; and the environmental signals that modulate circadian rhythms.

received

June 29, 2018

accepted after revision July 1,2018
Issue Theme Nutrition in Child Health Conditions; Guest Editor: Pilar Codoñer Franch, MD, PhD

DOI https://doi.org/ 10.1055/s-0038-1670667. ISSN 2474-5871.
In the second part, the concept of chronodisruption, or the impairment of circadian rhythms, is described. The causes are introduced, as well as the evidence suggesting the deleterious effects of this condition on nutrition and metabolism, especially during childhood.

This review aims to highlight the importance of the life habits that should be adapted to the organism's circadian rhythm to prevent metabolic disorders in children.

\section{Circadian Rhythms: Structure and Regulation}

The importance of circadian rhythms is suggested by their high level of conservation in evolution. ${ }^{1}$ Moreover, in humans, numerous factors have been shown to display a circadian expression or activity. Endogenous circadian clocks regulate

\section{Copyright $\odot 2018$ Georg Thieme Verlag License terms KG Stuttgart · New York \\ (c) $(1) \$$}


a broad spectrum of biological functions via multiple routes. Circadian rhythms are cycles of gene expression, metabolism, and behaviors created by the internal clocks that govern a multitude of metabolic functions, such as hepatic lipid metabolism, cardiovascular function, obesity regulation, and glucose homeostasis. For instance, circadian rhythms influence circulating leptin, ghrelin, adiponectin, and PAI-1, as well as lipid metabolism and Ucp 1 in brown adipose tissue. ${ }^{2,3}$

The critical role of circadian rhythms is confirmed by the diversity of the mechanisms that maintain their structure and adaptability based on the environment.

\section{Central Clock}

The first of these mechanisms is commonly called the central clock, a "master" clock within the suprachiasmatic nucleus (SCN) of the anterior hypothalamus, that primarily coordinates the multiple, single-cell circadian oscillators, which, when synchronized, regulate overt rhythms of physiology and behavior. The SCN requires exogenous inputs to track environmental time and mainly responds to the light-dark cycle.

Similar clock oscillators, sometimes termed "slave" clocks, have been found in peripheral tissues, such as the liver, intestines, heart, and retina.

The mechanisms through which the SCN synchronizes peripheral clocks are complex and not yet fully understood. Neuroendocrine pathways are likely to play a key role in this process because the autonomous nervous system and hormones, such as pineal melatonin and adrenal glucocorticoids, are under SCN control.

\section{Melatonin}

The central clock is tightly linked to the control of the expression of melatonin, the main circadian hormone. Melatonin, or $\mathrm{N}$-acetyl-5-methoxytryptamine, is synthesized by the pineal gland and is secreted exclusively at night, under the tight control of the SCN clock. Melatonin is synthesized from the amino acid tryptophan, which is converted into 5hydroxytryptophan by tryptophan hydroxylase before being decarboxylated into serotonin. From serotonin, two major enzymatic steps are involved. The first step is $\mathrm{N}$-acetylation by arylalkylamine $N$-acetyltransferase to yield $N$-acetylserotonin. The second step is the transfer of a methyl group from 5 -adenosylmethionine to the 5-hydroxy group of $\mathrm{N}$-acetylserotonin to yield melatonin. This reaction is catalyzed by hydroxyindole-O-methyltransferase.

All vertebrate species, regardless of their specific circadian activity pattern, experience elevated pineal melatonin production and secretion at night, with minimal synthesis during the day. In the morning, the human eye perceives blue light and sends a stimulus to the central clock in the SCN. The SCN relays this information to the pineal gland, where melatonin is produced. In response to this signal, the expression of arylalkylamine $\mathrm{N}$-acetyltransferase, the first enzyme necessary for melatonin production, is inhibited, preventing melatonin production. In the evening, sunlight is composed of more orange light than blue light, stimulating the production of melatonin. ${ }^{4}$
As a major hormonal output, the daily rhythm of plasma melatonin distributes temporal cues generated by the SCN to the multitude of tissue targets expressing melatonin receptors. Once produced, melatonin enters the bloodstream and reaches its receptors, MT1 and MT2. ${ }^{5,6}$ These ones are G proteincoupled receptors that are present at the surface of most human cells, such as the $\beta$ cells of the pancreas and adipocytes between others. The central clock, via melatonin expression, communicates a circadian signal to the rest of the organism (-Fig. 1). As a result, body temperature and blood pressure are adapted, favoring the start of sleep. This hormone is therefore a determinant modulator of the sleep/wake schedule.

In human studies, it is possible to analyze the central clock circadian rhythm by the quantification of melatonin, which can be done in body fluids such as saliva, blood, breast milk, or urine. ${ }^{7}$

The requirement of melatonin for the maintenance of a robust circadian rhythm is even evident in the peripheral oscillator of the adrenal cortex, which is strongly influenced by the rhythm of the adrenocorticotropic hormone. The cortisol peak (opposed to melatonin) is produced in the morning in response to adrenal activation by the adrenocorticotropic hormone, which is under SCN control.

\section{Clock Genes}

In mammals, the clock is an intracellular mechanism sharing the same molecular components in SCN neurons and peripheral cells. However, the circadian rhythms in the human body are not uniquely regulated by the central clock. Independent of melatonin action, human tissues display circadian patterns of gene expression. This phenomenon is due to the clock genes, or molecular oscillators, which are a group of transcription factors and inhibitors forming a double regulation-loop mechanism that lasts $\sim 24$ hours. $^{8}$ The proteins coded in the genes Clock, Bmal1, Period (Per1, Per2 and Per3), Cryptochrome (Cry1 and Cry2), and Rev-Erbo are periodically expressed or activated (-Fig. 2). This mechanism, which has been evidenced in vivo, is also maintained in cell cultures; therefore, it is sufficient to confirm a circadian pattern of the expression of genes. In addition, it regulates the expression of the clockcontrolled genes associated with cell metabolism, explaining the periodic expression of numerous metabolic factors. The integrity of the activity of the clock genes appears to be essential for maintaining metabolic homeostasis. Polymorphisms of Clock and Cry1 have been associated with abdominal obesity. ${ }^{9}$ Moreover, a polymorphism of the gene Clock has been associated with increased incidence of type II diabetes. ${ }^{10}$

The expression of clock genes structures circadian rhythms in the organs. Collectively with the genes they regulate, they constitute the peripheral clocks.

\section{Zeitgebers}

Together, the central clock and the peripheral clocks structure the circadian rhythms of an organism. Nevertheless, the human body is continuously exposed to numerous environmental changes. Different mechanisms allow the organism 


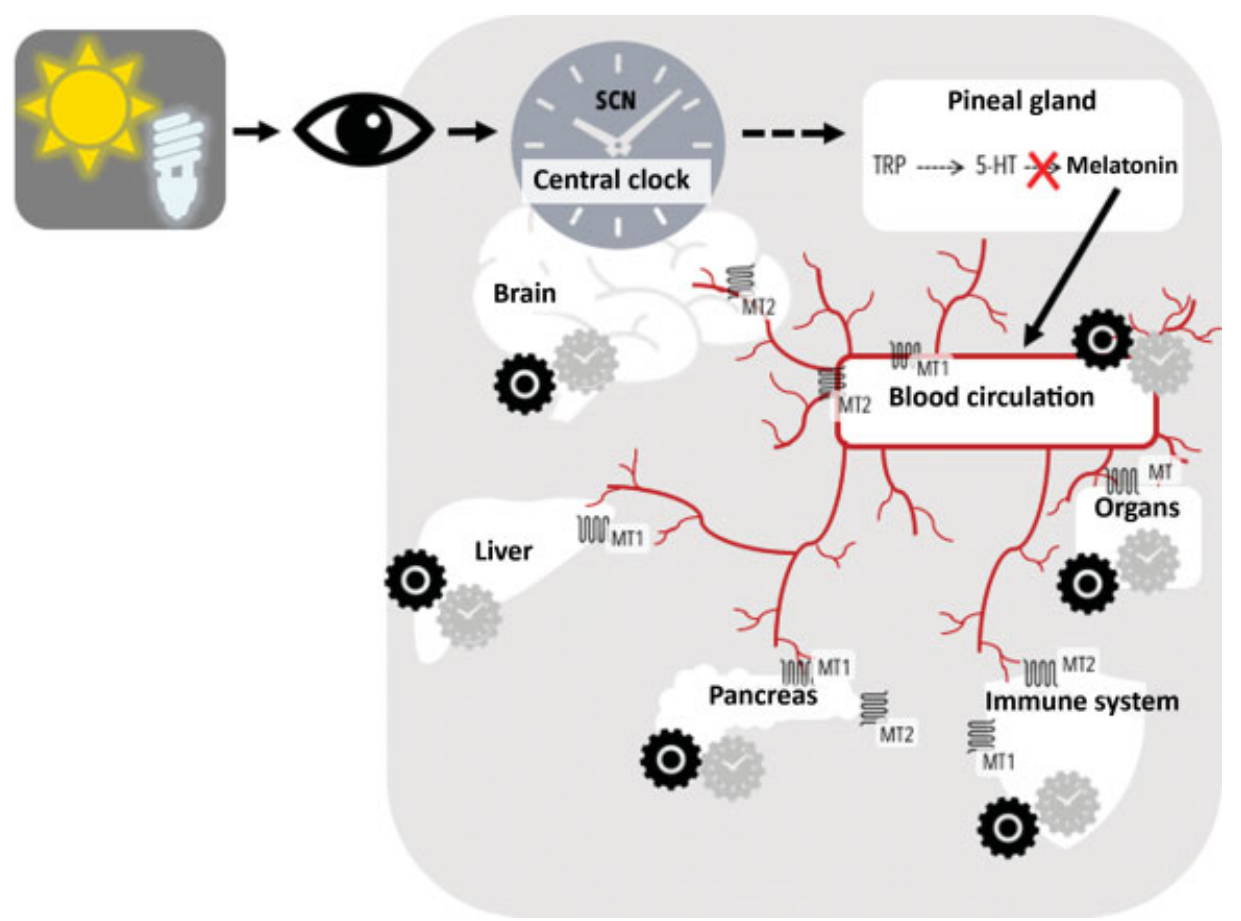

Fig. 1 Melatonin, the principal circadian hormone. The human retina perceives environmental light and converts it into a neuronal signal that is sent to the central clock, located in the suprachiasmatic nucleus (SCN) of the hypothalamus. The central clock relays the message to the pineal gland to control melatonin production. In the pineal gland, tryptophan (TRP) is converted into serotonin (5-HT). At night, the enzyme arylalkyl $\mathrm{N}$-acetyltransferase (NAT) catalyzes one of the two chemical reactions necessary to transform serotonin into melatonin. During the day, the light signal inhibits NAT synthesis and melatonin is not produced. This cyclic inhibition is responsible for the circadian variation in melatonin levels in the bloodstream. Melatonin's activity in the body is mediated by its binding to its receptors, MT1 and MT2, which are G protein-coupled receptors that have been identified on the surface of cells of numerous organs. ${ }^{6}$

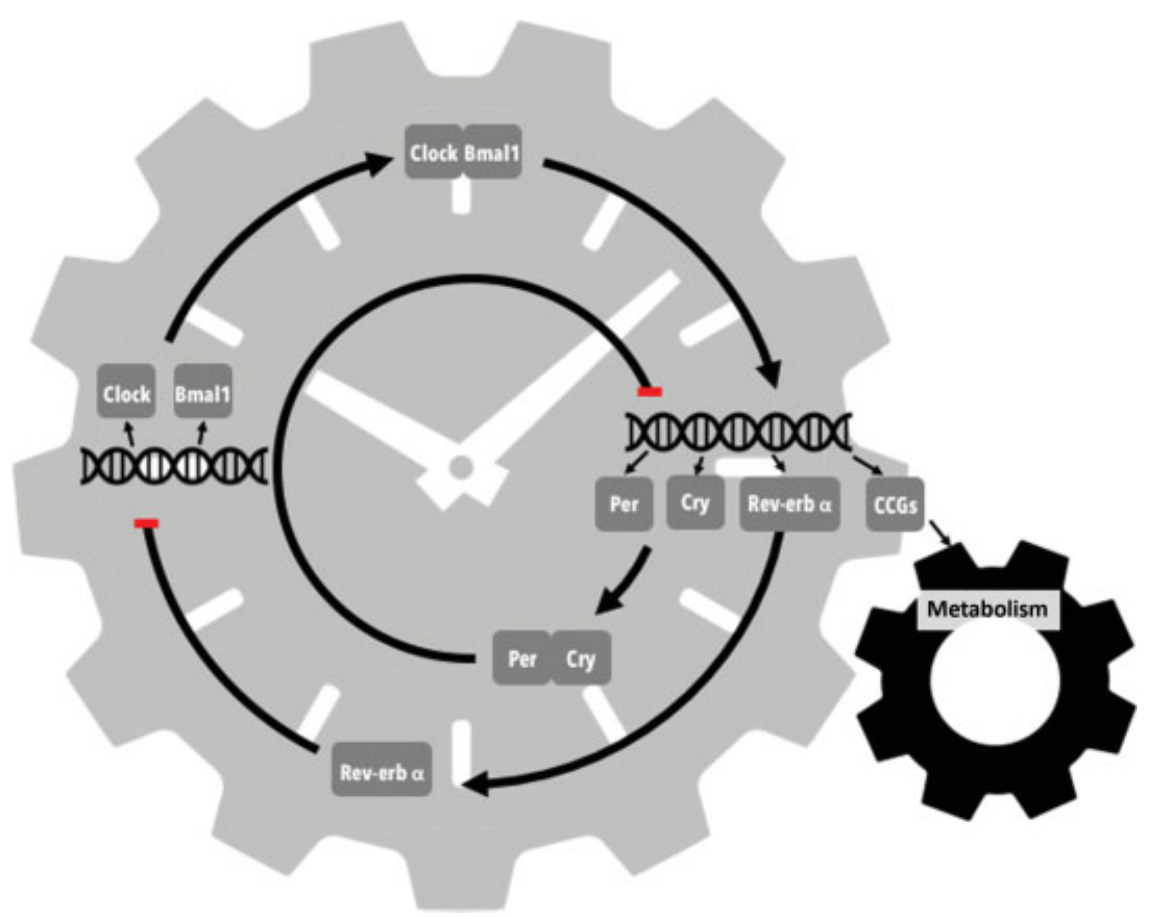

Fig. 2 Schematic circadian expression of clock genes. The expression of clock genes is a double regulation-loop mechanism that lasts $\sim 24$ hours. It begins with the expression and heterodimerization of the genes Clock and Bmal1, which activate the expression of the genes Per, Cry, Rev-Erba, and the clock-controlled genes related to metabolism. Rev-Erba inhibits Bmal1 expression, and therefore prevents dimerization with Clock. At the same time, Per and Cry accumulate, forming a complex and inhibiting the expression of Per, Cry, Rev-Erba, and the CCGs. Rev-Erba levels drop, allowing Bmal1 expression. New Bmal1-Clock complexes are formed, and another subjective day begins. 
to detect and adapt to those changes. The external elements impacting circadian rhythms are referred to as zeitgebers, meaning time giver or synchronizer in German. The daily light/dark cycle is the most frequently described zeitgeber, but several studies have underlined the importance of the following parameters: external temperature, feeding schedule, social interaction, sleeping schedule, and physical activity. ${ }^{11}$ Indeed, these elements impact circadian rhythms through diverse pathways and should be taken into consideration as much as possible in human studies.

\section{Light Exposure}

First, the environmental blue light, detected by the retina, influences the melatonin expression pattern (-Fig. 1). Humans are naturally exposed to blue sunlight in the morning. However, currently humans are also exposed to blue light from artificial sources for the rest of the day.

\section{Physical Activity}

Physical activity produces a modification of energy expenditure and therefore induces a metabolic adaptation. The nature and duration of the effort will mobilize resources through the activation of different metabolic pathways and hormonal actions that logically impact the basal circadian metabolism. $^{12}$

\section{Sleeping Schedule and Patterns}

It is well known that sleep is essential for health, particularly during childhood. Many reparative mechanisms occur during sleep. Endocrine activity adapts to the decreased physical activity, and there is a peak of growth hormone. ${ }^{13}$ Sleeping patterns can be affected by many factors, including age; the amount of recent sleep or wakefulness; the time of the day or night relative to the individual's internal clock; other behaviors prior to sleep, such as exercise; stress; environmental conditions, such as temperature and light; and various chemicals.

A regular sleeping schedule, adapted to the children's needs and in coherence with the children's central clock, is critical for healthy development.

Furthermore, it has been observed that children who go to bed late tend to eat more and later, ${ }^{13}$ showing the association between the sleeping schedule and the feeding schedule, another essential zeitgeber.

\section{Feeding Schedule and Composition}

The feeding schedule plays a role in the regulation of metabolic circadian rhythms. Before the beginning of a meal until the end of digestion, the body makes metabolic adaptations to process the nutrients. The nature and amplitude of the metabolic reactions vary according to the caloric intake, the nutritional composition, and, very importantly, the time of the intake. However, the last parameter is often ignored. The digestion process itself, along with the associated production of metabolic hormones and enzymes, modulates the availability of nutrients in the blood and impacts the internal clocks. Even before eating, the organism anticipates the meal based on the feeding schedule of the previous days and starts the secretion of hormones and factors to facilitate the digestion. This food anticipatory activity is considered a circadian rhythm. ${ }^{8}$ Thus, the feeding schedule has an undeniable importance in circadian rhythm modulation.

Several studies have shown that meal composition also has a great role to play. For instance, in mice, the modification of fat, protein, and carbohydrate content in the diet impaired the expression of clock genes in the liver and kidneys. ${ }^{8}$ More investigations are necessary to identify the molecular pathways involved in the regulation of circadian clocks by specific nutrients.

Food composition could also participate in the regulation of circadian rhythm by the exogenous contribution of melatonin. During the first weeks of life, the newborn does not produce melatonin but absorbs it from the intake of breast milk. After 6 months of life, melatonin is produced from the essential amino acid tryptophan, which is drawn from circulation by the pineal gland. Nevertheless, an appreciable amount of melatonin is still derived from food in childhood, because melatonin is present in numerous nutrients, especially nuts. Even if there are important differences in melatonin bioavailability in the adult population (from 1 to $37 \%$ ), dietary intake appears to modulate circadian rhythms. Moreover, exogenous melatonin is currently prescribed to improve insomnia or jet lag and seems to facilitate sleep initiation. $^{14}$

This wide array of elements participates in the modulation of circadian rhythms, and there are several pathways coupling the molecular clock to metabolism. Furthermore, it appears that feeding time has a dramatic effect on health. In addition to the amount and content of food, the time of ingestion is critical.

\section{Chronotypes}

Human preferences in the timing of sleeping and waking (called "chronotypes") are, at least partly, based on genetics, behavioral components, and light exposure. In the human population, different profiles of habits exist, which are associated with the chronotype of the individual. Even in the same family, one individual can display a morning chronotype, while his sibling displays an evening chronotype. The differences between chronotypes are characterized by several parameters. A morning chronotype feels more alert and ready to focus, work, or exercise in the morning. On the other hand, in an evening chronotype, those elements are delayed. Some studies have evidenced that in evening chronotype individuals, there is a delay of $\sim 2$ hours in the expression of the clock genes Per 3 and $\mathrm{Nr} 1 \mathrm{~d} 2$, in melatonin onset and in sleep timing, compared with extreme morning chronotype individuals. ${ }^{15,16}$ Chronotypes have been assessed mainly by questionnaires that are designed to associate individuals with tendencies (morningness-eveningness). ${ }^{17}$ The use of these questionnaires is a useful method to identify an individual chronotype. Most of the population belongs to an intermediary chronotype. ${ }^{18}$ Evening chronotype people have a natural tendency to intake greater energy apart from main meals and also reduced physical activity. ${ }^{19}$ The chronotype influences the habits, 


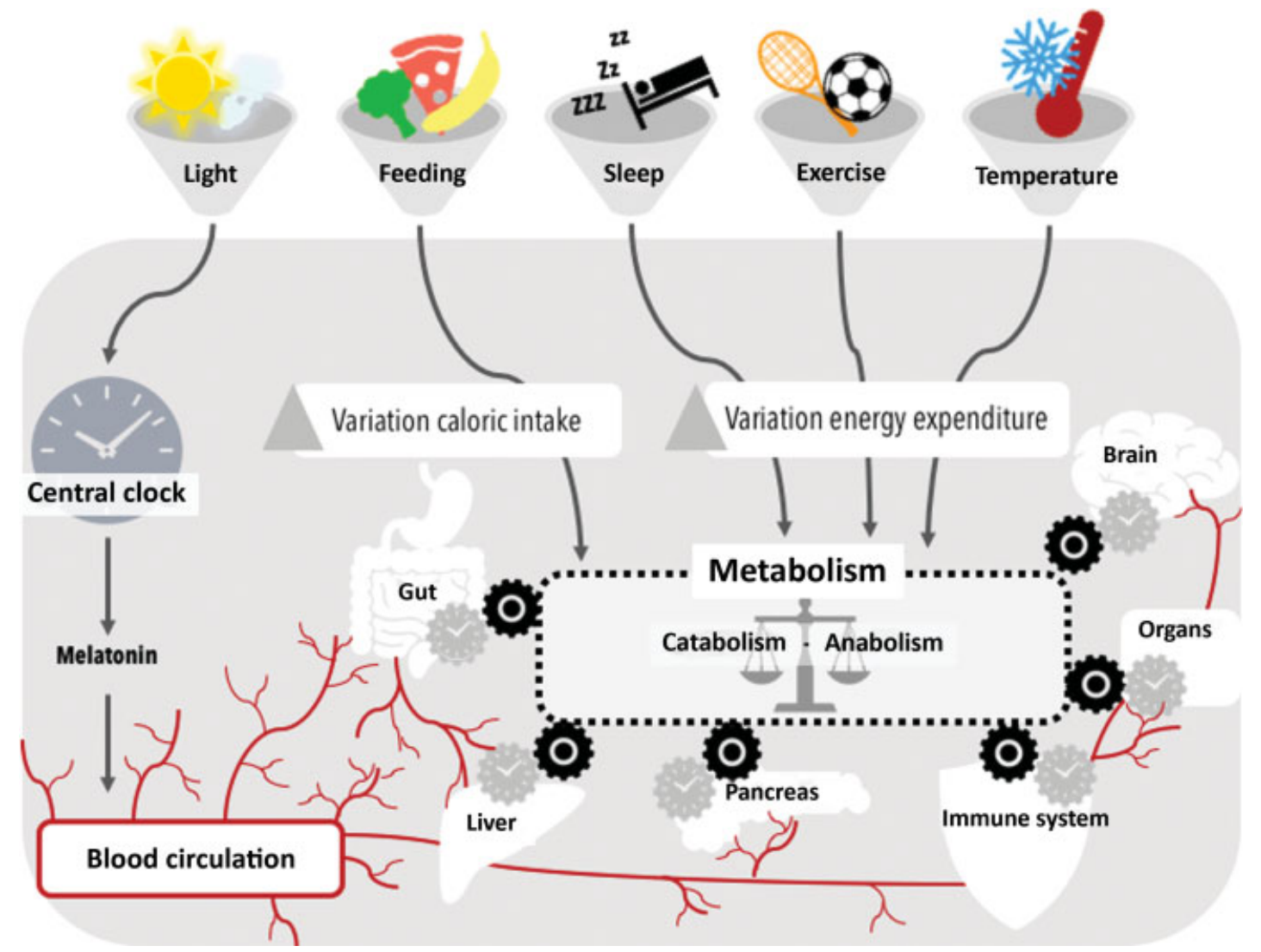

Fig. 3 Influence of zeitgebers on circadian metabolism. Upper part of the figure: the inputs, zeitgebers, and external parameters influenced by life habits that participate in circadian modulation of metabolism. Light influences the central clock and melatonin production. Feeding composition and schedule; sleep schedule, duration, and quality; exercise and environmental temperature influence the variations in caloric intake and energy expenditure of the organism. This will induce metabolic changes and adaptations in the involved organs. Two situations exist: The inputs received by the organism are coherent; therefore, the central clock and the peripheral clocks are synchronized. The catabolic and anabolic reactions are adapted to the individual's lifestyle and activities. The metabolism is optimized. Incoherent messages are perceived by the different organs leading to desynchronization of the different clocks. The organism fails to adapt to contradictory signals. This condition of disrupted circadian rhythms is called chronodisruption. Metabolic homeostasis is consecutively impaired, leading to disorders such as diabetes, obesity, and cardiovascular diseases.

and the habits modulate the signals received by the body and induce metabolic adaptations (-Fig. 3). When those signals are coherent and synchronized with the internal clocks, the metabolism is optimized. In the opposite situation, a relevant alteration of physiological and behavioral circadian rhythms, in which a change in normal melatonin secretion occurs, is defined as a "chronodisruption" or "impairment of circadian rhythms." In this case, the different metabolic processes lack coordination, and, if this lasts for a long time or occurs frequently, damages can be induced. This impairment is critical during childhood, because the period of growth and development may be affected. Furthermore, it has been shown that chronodisruption favors the development of an obesogenic environment.

\section{Chronodisruption: Causes and Consequences}

As described in - Fig. 3, the organism perceives environmental signals that arrive at the central clock. These signals are then sent via neuronal connections or circulating humoral factors to peripheral clocks located in several organs to prevent the dampening of circadian rhythms in these tissues. The circadian clock is intimately connected to the metabolism. ${ }^{20-22}$ From a molecular point of view, it has been shown that the clock machinery controls the expression of essential genes within numerous metabolic pathways.

Chronodisruption can be caused by a lack of coordination between the different elements mentioned, and several studies (experimental and clinic) have underlined the health consequences of chronodisruption. Studies in mice indicate that changes in feeding schedule carry clear metabolic implications. Mice housed in constant bright or dim light consumed more food during the subjective light phase and exhibited significantly increased body mass and reduced glucose tolerance. ${ }^{23}$ The metabolic effects of feeding schedule are highlighted by a decrease in hepatic triglyceride content with restricted nighttime feeding with regular chow without modification of total daily caloric consumption. ${ }^{24}$

Sleeping time and duration not only affect the amount of light that the organism will perceive but also impact the energy expenditure, meal schedule and, therefore, metabolic homeostasis. Different studies have aimed to characterize the recommended sleeping habits, especially during childhood. 
There is evidence of a link between short sleep duration and obesity severity as well as cardiovascular risk. In a European study, a sleep duration shorter than 8 hours was associated with higher adiposity markers in teenagers, ${ }^{25}$ and we have observed that obese children with shorter sleep duration showed more severe obesity and higher arterial pressure. $^{13}$

Interestingly, other studies have demonstrated that even more than sleep duration, the sleeping schedule impacts metabolism. For example, in teenagers, it was evidenced that, for the same sleep duration, individuals who were going to sleep and waking up later were more overweight. ${ }^{18}$

Similarly, in adults, nurses working the night shift displayed chronodisruption, evidenced by delayed melatonin secretion, and demonstrated an increased tendency toward weight gain, obesity, and comorbidities such as diabetes. ${ }^{26}$

Other habits, such as artificial light exposure, especially blue light, ${ }^{11}$ during the 2 hours before sleep, induce a delay in melatonin expression. In US university students, artificial light exposure during the 2 hours before sleep has been associated with later and shorter sleeping times ${ }^{27}$ and can induce chronodisruption and its associated metabolic impairments.

These results suggest that avoiding blue light exposure during the 2 hours before sleep, going to bed early, and sleeping a sufficient amount of time play a protective role in metabolic homeostasis and is recommendable to prevent metabolic disturbances, especially during childhood.

As mentioned earlier, physical exercise increases energy expenditure, inducing a modification in metabolism. The impact of physical activity on circadian rhythms has been investigated in adults. A reduced and delayed (90 minutes) wrist temperature amplitude peak was observed in women running 45 minutes in the evening compared with in the morning. ${ }^{12}$ This delay in circadian temperature suggests the modification of circadian rhythms at the global level of the organism. If these features are confirmed by future research, practicing sports earlier than the 2 hours before sleep should be advised to avoid a delay in melatonin expression, as well as avoiding late sleep and meals.

Finally, the schedule and quality of nutrient intake at the different meals impacts metabolism as well. The following research assessed the importance of feeding schedule on metabolism to identify the habits that lead to a better metabolization of nutrients.

A study compared a group of individuals undergoing two different conditions: either eating their main meal early (14:00) or late (17:30). A delay in circadian rhythm was measured in the late main meal condition. Additionally, during this condition, participants displayed decreased diversity in mouth microbiota (diversity in microbiota being a protective parameter), an increase in weight gain and poorer metabolic indicators. ${ }^{28}$ Similarly, in a study conducted in individuals following a restricted diet, the group with the ingestion of a higher caloric intake during breakfast demonstrated greater weight loss than the group that consumed a high-calorie dinner. ${ }^{29}$

The influence of food schedule on metabolic parameters has been emphasized in several studies. Triglyceride and free fatty acid blood levels were lower after the first meal of the day compared with the levels after the second and third meals. $^{3}$

Although there was no delay in melatonin expression, an effect on clock gene expression was noticed. For instance, switching to eating 5 hours later delayed the Per2 gene expression. ${ }^{8}$ This is consistent with the hypothesis that meal schedules alter the peripheral clock more than the central clock.

All together, these results suggest that consuming the highest caloric meal early in the day and respecting a daily fasting period constitute a healthy lifestyle adapted to circadian clocks and the associated metabolism.

\section{Key Questions that Remain Unanswered}

In recent years, interest in the link between circadian rhythms and metabolism has increased, and many elements have already demonstrated the importance of this topic. In children, the adoption of healthy habits to maintain an adequate coordination with circadian rhythms is highly desirable. Nevertheless, many questions remain unanswered.

First, the mechanisms of the peripheral and central clocks are not fully understood. Melatonin's action and its effects on different targeted mechanisms should be investigated at a molecular level. Likewise, the pathways modulated by its expression should be identified.

Second, the regulation of the clock genes by external elements, namely nutrition, remains unknown.

The identification of gene polymorphisms in the central and peripheral clocks associated with metabolic parameters is a strategy to understand the implications of these mechanisms in health.

Moreover, the evaluation of the age-specific effects of these mechanisms is particularly important considering the major changes in weight, growth hormones, and steroid hormones that occur at different ages. Childhood is a key moment in which the importance of circadian rhythm should be investigated, because the organism is in a state of constant change and evolution. Human growth is a complex process, with variations in growth rates occurring in the short-term over weeks as well as seasonally. The growth rate is greatest during the summer season, suggesting a relationship with day length and, consequently, with light. Indeed, during growth phases, metabolic variations and impairments are likely to have long-term impacts.

Furthermore, circadian rhythms are not only important in physiological states, as several pathologies have been related to disturbed lifestyle habits.

Future studies should evaluate how life habits influence circadian metabolic variations to determine the best schedule to adapt to the metabolic circadian rhythms. For instance, blue light exposure and physical exercise should be limited during the 2 hours before sleep to facilitate an early bedtime. An early sleeping and feeding schedule is associated with a healthier metabolic status, and several studies have demonstrated a better ability of the organism to digest nutrients efficiently during the early part of the day. Considering the results mentioned in the present review, this adaptation of life habits, 
in synchronization with the metabolism, would have a protective effect against metabolic disorders.

Finally, the implications of interindividual differences, such as belonging to a certain chronotype, should be better understood in order take them into consideration for personalized schedules and variations in nutrition composition during the day. On a large scale, a more accurate understanding of the impact of circadian rhythms on health, especially during childhood, will amplify the relevance of prevention campaigns regarding healthy life habits.

\section{Conclusion}

Circadian rhythms are the changes in biological processes that occur on a daily basis; among these changes are the reactions involved in metabolic homeostasis. Circadian rhythms are structured by the central clock in the SCN of the hypothalamus via the control of melatonin expression and by the peripheral clocks, which are intracellular mechanisms composed of the "clock genes" whose expression follows a circadian pattern. Circadian rhythms are impacted by signals from the environment: the zeitgebers or time givers. These signals include light exposure, feeding schedule and composition, sleeping schedule and pattern, temperature, and physical exercise. Metabolism is optimized when the different clocks receive coherent signals synchronized with the internal clocks. The opposite situation is defined by the term chronodisruption. In this context, metabolic homeostasis is impacted. Chronodisruption contributes to an increased risk of metabolic disorders, including obesity, from a very young age, but this increased risk is preventable. Recent research suggests that, in addition to the incitation to improve diet quality, to reduce caloric intake, and to increase physical exercise, prevention campaigns must include advice for schedules that are adapted to the internal clocks. Further research, especially focusing on children, will help to develop more accurate prevention campaigns to favor the healthy growth and development of children.

\section{Conflict of Interest}

None.

\section{References}

1 Feng D, Lazar MA. Clocks, metabolism, and the epigenome. Mol Cell 2012;47(02):158-167

2 Bass J, Takahashi JS. Circadian integration of metabolism and energetics. Science 2010;330(6009):1349-1354

3 van den Berg R, Kooijman S, Noordam R, et al. A diurnal rhythm in brown adipose tissue causes rapid clearance and combustion of plasma lipids at wakening. Cell Reports 2018;22(13):3521-3533

4 Jenwitheesuk A, Nopparat C, Mukda S, Wongchitrat P, Govitrapong $\mathrm{P}$. Melatonin regulates aging and neurodegeneration through energy metabolism, epigenetics, autophagy and circadian rhythm pathways. Int J Mol Sci 2014;15(09):16848-16884

5 Claustrat B, Leston J. Melatonin: physiological effects in humans. Neurochirurgie 2015;61(2-3):77-84

6 Slominski RM, Reiter RJ, Schlabritz-Loutsevitch N, Ostrom RS, Slominski AT. Melatonin membrane receptors in peripheral tissues: distribution and functions. Mol Cell Endocrinol 2012;351 (02):152-166
7 Cohen Engler A, Hadash A, Shehadeh N, Pillar G. Breastfeeding may improve nocturnal sleep and reduce infantile colic: potential role of breast milk melatonin. Eur J Pediatr 2012;171(04):729-732

8 Potter GDM, Cade JE, Grant PJ, Hardie LJ. Nutrition and the circadian system. Br J Nutr 2016;116(03):434-442

9 Ye D, Cai S, Jiang X, et al. Associations of polymorphisms in circadian genes with abdominal obesity in Chinese adult population. Obes Res Clin Pract 2016;10(Suppl 1):S133-S141

10 Corella D, Asensio EM, Coltell O, et al. CLOCK gene variation is associated with incidence of type- 2 diabetes and cardiovascular diseases in type-2 diabetic subjects: dietary modulation in the PREDIMED randomized trial. Cardiovasc Diabetol 2016;15:4

11 Bonmati-Carrion MA, Arguelles-Prieto R, Martinez-Madrid MJ, et al. Protecting the melatonin rhythm through circadian healthy light exposure. Int J Mol Sci 2014;15(12):23448-23500

12 Rubio-Sastre P, Gómez-Abellán P, Martinez-Nicolas A, Ordovás JM, Madrid JA, Garaulet M. Evening physical activity alters wrist temperature circadian rhythmicity. Chronobiol Int 2014;31(02): 276-282

13 Navarro-Solera M, Carrasco-Luna J, Pin-Arboledas G, GonzálezCarrascosa R, Soriano JM, Codoñer-Franch P. Short sleep duration is related to emerging cardiovascular risk factors in obese children. J Pediatr Gastroenterol Nutr 2015;61(05):571-576

14 Meng X, Li Y, Li S, et al. Dietary sources and bioactivities of melatonin. Nutrients 2017;9(04):367

15 Ferrante A, Gellerman D, Ay A, et al. Diurnal preference predicts phase differences in expression of human peripheral circadian clock genes. J Circadian Rhythms 2015;13:4

16 Paine SJ, Gander PH. Differences in circadian phase and weekday/ weekend sleep patterns in a sample of middle-aged morning types and evening types. Chronobiol Int 2016;33(08):1009-1017

17 Horne JA, Ostberg O. A self-assessment questionnaire to determine morningness-eveningness in human circadian rhythms. Int J Chronobiol 1976;4(02):97-110

18 Urbán R, Magyaródi T, Rigó A. Morningness-eveningness, chronotypes and health-impairing behaviors in adolescents. Chronobiol Int 2011;28(03):238-247

19 Olds TS, Maher CA, Matricciani L. Sleep duration or bedtime? Exploring the relationship between sleep habits and weight status and activity patterns. Sleep 2011;34(10):1299-1307

20 Asher G, Schibler U. Crosstalk between components of circadian and metabolic cycles in mammals. Cell Metab 2011;13(02):125-137

21 Bass J. Circadian topology of metabolism. Nature 2012;491 (7424):348-356

22 Eckel-Mahan K, Sassone-Corsi P. Epigenetic regulation of the molecular clockwork. Prog Mol Biol Transl Sci 2013;119:29-50

23 Fonken LK, Workman JL, Walton JC, et al. Light at night increases body mass by shifting the time of food intake. Proc Natl Acad Sci U S A 2010;107(43):18664-18669

24 Adamovich Y, Rousso-Noori L, Zwighaft Z, et al. Circadian clocks and feeding time regulate the oscillations and levels of hepatic triglycerides. Cell Metab 2014;19(02):319-330

25 Garaulet M, Ortega FB, Ruiz JR, et al. Short sleep duration is associated with increased obesity markers in European adolescents: effect of physical activity and dietary habits. The HELENA study. Int J Obes 2011;35(10):1308-1317

26 Peplonska B, Bukowska A, Sobala W. Association of rotating night shift work with BMI and abdominal obesity among nurses and midwives. PLoS One 2015;10(07):e0133761

27 Orzech KM, Grandner MA, Roane BM, Carskadon MA. Digital media use in the $2 \mathrm{~h}$ before bedtime is associated with sleep variables in university students. Comput Human Behav 2016;55(A):43-50

28 Collado MC, Engen PA, Bandín C, et al. Timing of food intake impacts daily rhythms of human salivary microbiota: a randomized, crossover study. FASEB J 2018;32(04):2060-2072

29 Garaulet M, Gómez-Abellán P. Timing of food intake and obesity: a novel association. Physiol Behav 2014;134:44-50 